\title{
Research of the mixing process in vortex static type devices
}

\author{
Vitaliy Afanasenko ${ }^{1, *}$ and Petr Kulakov ${ }^{1}$ \\ ${ }^{1}$ Ufa State Petroleum Technological University, 1, Kosmonavtov St. , Ufa, 450062, Russia
}

\begin{abstract}
Mixing in chemical technology is used to intensify chemical and heat-mass transfer processes, as well as for preparing emulsions, suspensions and obtaining homogeneous systems. The simplest way to carry out the process, which does not require an additional supply of mechanical energy, is to mix in a direct-flow environment, which only requires flow energy. Analysis of the existing designs of direct-flow mixers showed that the use of swirling devices of various types in order to increase the efficiency of the process is very promising. This article describes a study of the influence of the geometrical dimensions of the screw on the efficiency of mixing a two-component mixture using the FlowVision software package.
\end{abstract}

\section{Introduction}

Mixing in liquid media is widely used in industry for the preparation of emulsions, suspensions and the preparation of homogeneous systems (solutions), as well as for the intensification of chemical, thermal and diffusion processes. In the latter case, mixing is carried out directly in devices designed for carrying out these processes, equipped with mixing devices [1-4].

The purpose of mixing is determined by the purpose of the process. When preparing emulsions for intensive crushing of the dispersed phase, it is necessary to create significant shear forces in a mixed medium, depending on the velocity gradient. In those areas of the apparatus where the fluid velocity gradient is of the greatest importance, the most intense fragmentation of the dispersed phase occurs.

In the case of homogenization, preparation of suspensions, heating or cooling of a mixed homogeneous medium, the purpose of mixing is to reduce concentration or temperature gradients in the volume of the apparatus.

When mixing is used to intensify chemical, thermal, and diffusion processes in heterogeneous systems, better conditions are created for supplying the substance to the reaction zone, to the phase boundary, or to the heat exchange surface. An increase in the degree of turbulence of the system, achieved with stirring, leads to a decrease in the thickness of the boundary layer and an increase and continuous updating of the surface of the interacting phases. This causes a significant acceleration of the processes of heat and mass transfer.

\footnotetext{
* Corresponding author: afanasenko.v.g@yandex.ru
} 
The methods of mixing and the choice of equipment for its implementation are determined by the purpose of mixing and the state of aggregation of the materials being mixed. The processes of mechanical, pneumatic and circulating mixing require additional energy supply. Consider the simplest method of mixing - mixing the media in the pipelines, the process will occur in a direct flow, and only the flow energy is spent on it.

By analogy with mechanical mixers, the main factors characterizing the operation of direct-flow mixers can be attributed [5-8]:

- energy loss;

- intensity (degree) of mixing;

- mixing efficiency.

In the study, vortex mixers were considered, in which a single-auger screw was used as a twisting device.

\section{Research methods}

For the study, computer simulation was used with the application of the FlowVision v2.3 software package. This product is designed to calculate hydro- and gas-dynamic problems (in a wide range of Reynolds and Mach numbers in arbitrary three-dimensional areas [5,915].

The basic equations in the FlowVision software package are equations Navier - Stokes (together with the continuity equation). To close these equations, depending on the specific problem, additional relations can be used that describe the change in density, turbulent transport, etc. Sets of such relations together with the Navier - Stokes equations are called models. In our calculations, we used the $k-\varepsilon$ model of a turbulent flow of a viscous fluid with small changes in density at large Reynolds numbers. To determine the concentration, the convection-diffusion transport equation was solved.

\section{Study of the main characteristics of the mixer when changing the step of the swirl flow}

The main geometric dimensions of axial twisting devices made in the form of a screw include a twist step, therefore, an important role is played by the study of the influence of a screw step on the main characteristics of the mixer.

To study the influence of the screw pitch used in the mixing device on the quality of mixing, a twisting device located in a pipe with a diameter of $0.3 \mathrm{~m}$, the length of which is constant and equal to $0.75 \mathrm{~m}$, was considered. The speed of the incoming components (kerosene, water) was set equal to $1.0-0.5 ; 2.0-1.0$ and $4.0-2.0 \mathrm{~m} / \mathrm{s}$, respectively. We examined the screws in increments of 0.18 to $0.45 \mathrm{~m}$.

A general view of the calculation model is shown in Figure 1. 


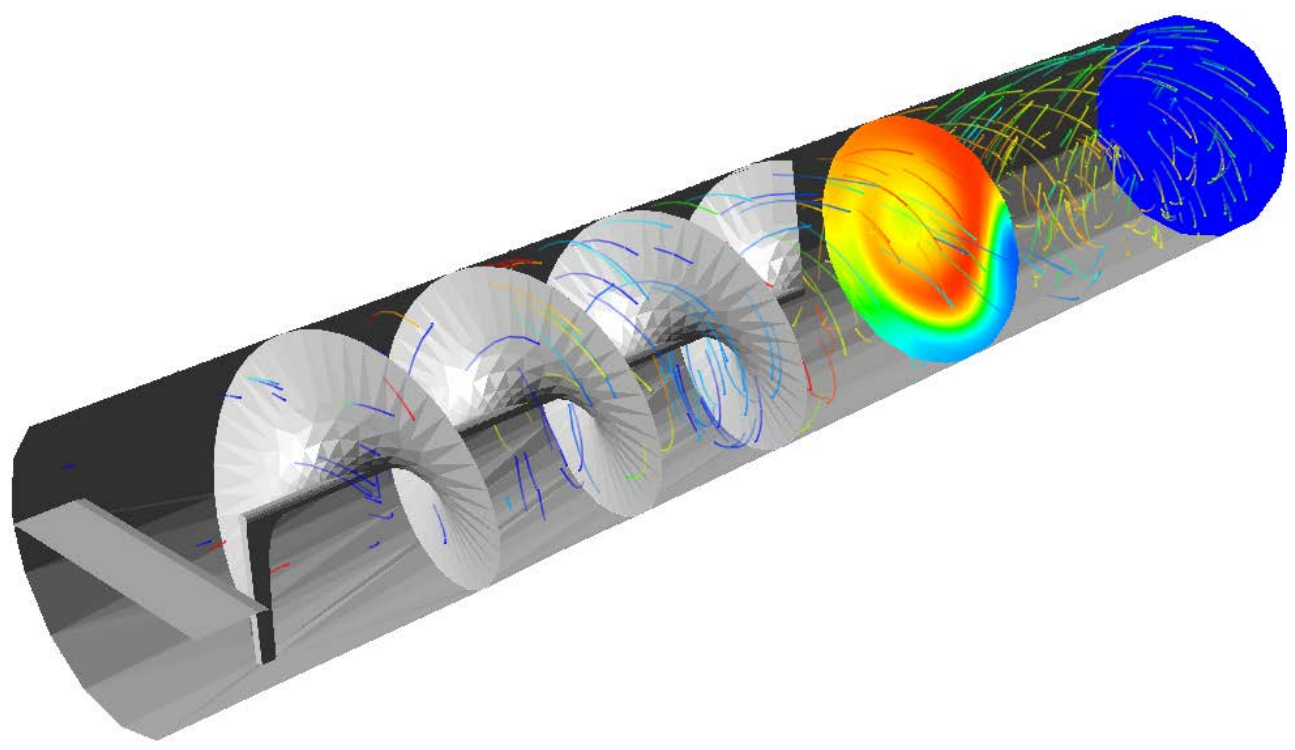

Fig. 1. The calculation model in general.

The concentration dispersion was estimated in the cross section of the pipe at a distance of $0.2 \mathrm{~m}$ from the twisting device. According to the data obtained, graphs of the dependence of the concentration dispersion in the plane perpendicular to the axis of rotation on the pitch of the twisting device were constructed (Figure 2).

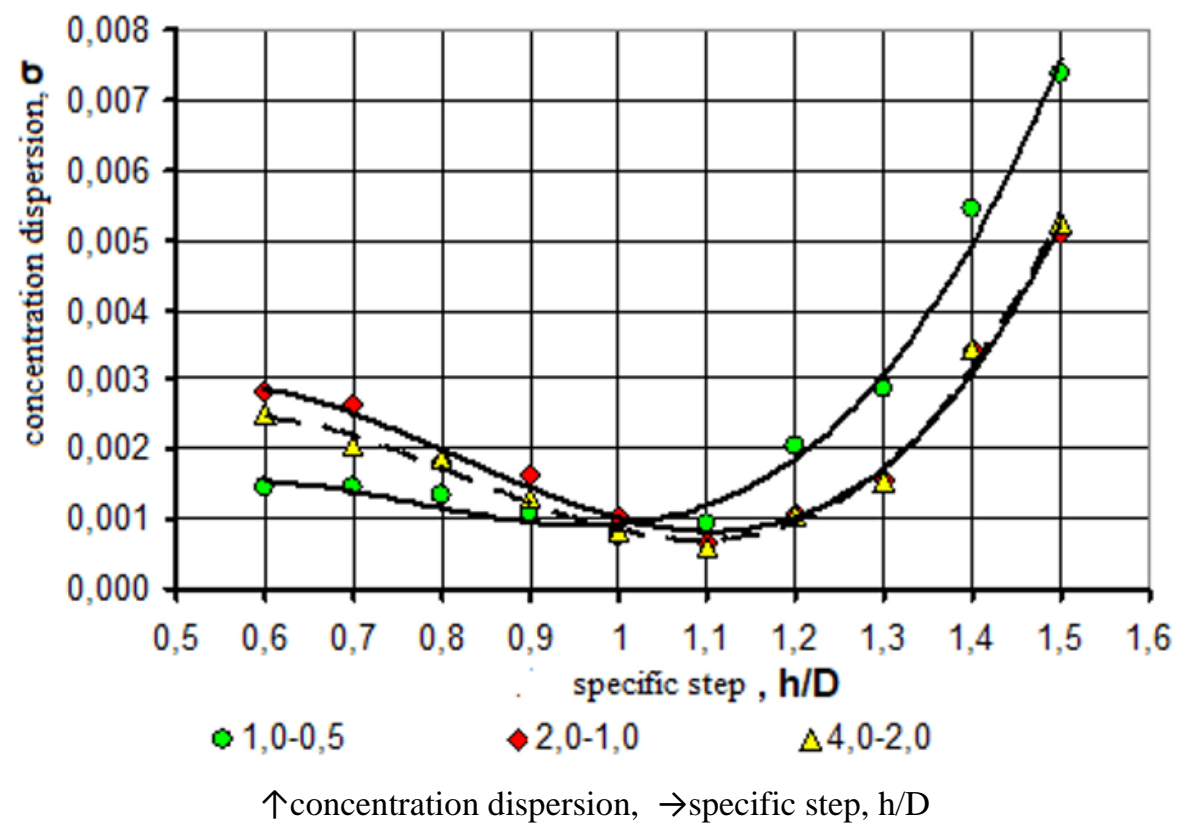

Fig. 2. Graph of the concentration dispersion versus the step length of the screw referred to the pipe diameter (h/D).

From the obtained dependence it follows that the quality of mixing with increasing screw pitch at the initial stage increases, but, reaching its maximum, begins to decrease. This is due to the fact that at a high intensity of the swirling flow, the process opposite to 
mixing occurs - separation of components in the field of action of centrifugal forces. This assumption is confirmed by the fact that with an increase in the flow velocity, the minimum dispersion of concentrations shifts to the right (to the region of a larger specific step). And with a small step (0.6-0.9), an increase in speed, leading to an increase in the flow swirl intensity, leads to a deterioration in the quality of mixing.

To study the dependence of the resistance, characterized by the pressure drop before and after the mixing device, on the step length of the screw, we considered the change in resistance in a circular pipe with a diameter of $0.3 \mathrm{~m}$, in which a screw of constant length $(0.5 \mathrm{~m})$ was installed. The velocities of the mixed components (kerosene and water) at the inlet were taken equal to: 1.0 and $0.5 \mathrm{~m} / \mathrm{s} ; 2.0$ and $1.0 \mathrm{~m} / \mathrm{s} ; 3.0$ and $1.5 \mathrm{~m} / \mathrm{s}$, and the average flow rates for the cases considered are $0.83,1.66$ and $2.49 \mathrm{~m} / \mathrm{s}$, respectively.

Based on the data obtained, we plotted the dependence of the pressure drop caused by the mixing device on the step length of the screw referred to the pipe diameter at various initial speeds, and also plotted the power-law trend lines (Figure 3).

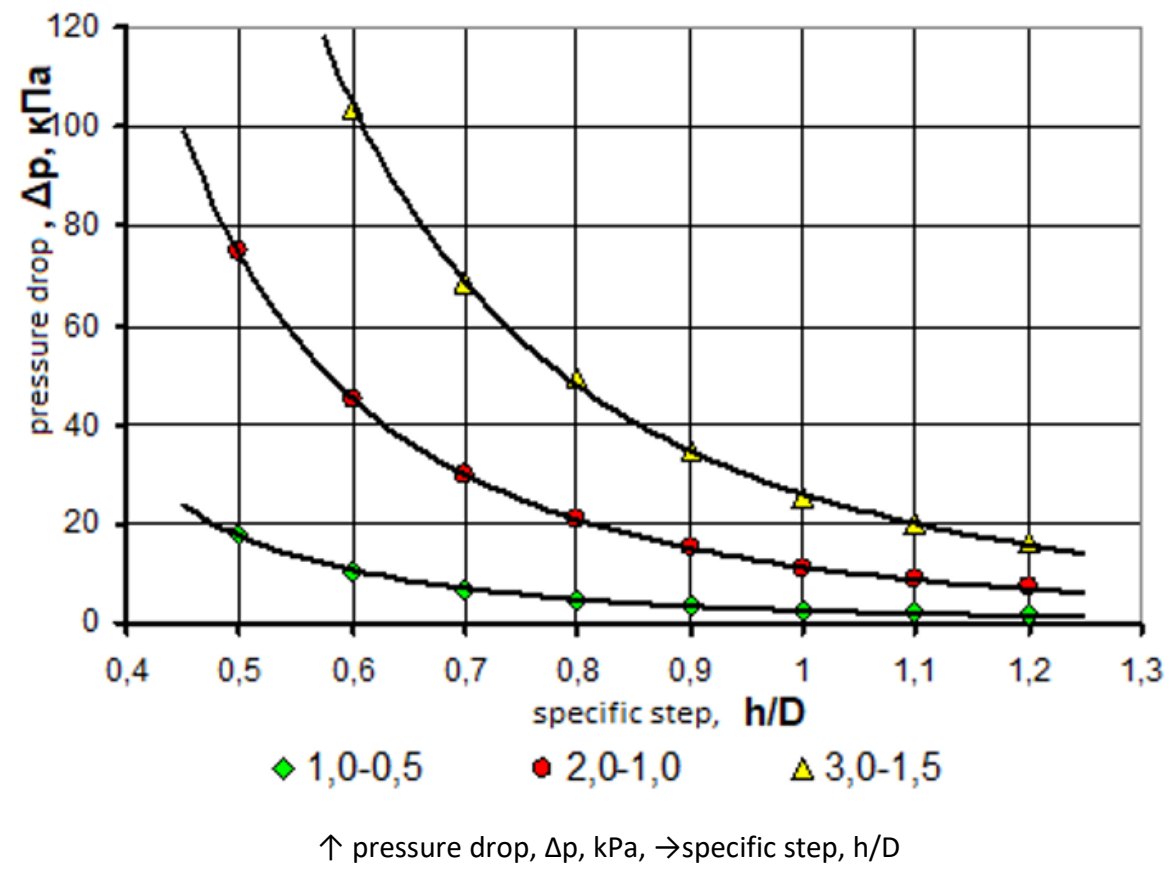

Fig. 3. Graph of the resistance of the mixing device $(\Delta \mathrm{p}, \mathrm{kPa})$ on the step length of the screw referred to the diameter of the pipe (h/D).

The obtained graphs can be described with a sufficient degree of accuracy by a powerlaw dependence of the following form:

$$
\Delta p=3,92 \cdot V_{c p}^{2,04} \cdot\left(\frac{h}{D}\right)^{-2,72}
$$

The average speed for a two-component mixture was determined by the law of conservation of momentum: 


$$
V_{c p}=\frac{\left(V_{\kappa 1}^{2} \cdot \rho_{\kappa 1} \cdot S_{\kappa 1}+V_{\kappa 2}^{2} \cdot \rho_{\kappa 2} \cdot S_{\kappa 2}\right) \cdot\left(S_{\kappa 1}+S_{\kappa 2}\right)}{\left(V_{\kappa 1} \cdot \rho_{\kappa 1} \cdot S_{\kappa 1}+V_{\kappa 2} \cdot \rho_{\kappa 2} \cdot S_{\kappa 2}\right) \cdot S_{m p}}
$$

where $V_{c p}$ - average flow rate, $\mathrm{m} / \mathrm{s}$;

$h$ - screw pitch, m;

$D$ - pipe diameter, m;

$V_{\kappa 1}, V_{k 2}$ - initial velocity of components, $\mathrm{m} / \mathrm{s}$;

$\rho_{\kappa 1}, \rho_{k 2}$ - the density of the mixed components, $\mathrm{kg} / \mathrm{m}^{3}$;

$S_{\kappa 1}, S_{k 2}, S_{m p}$ - pass-through areas of inlet pipes and pipes, $\mathrm{m}^{2}$.

\section{Study of changes in concentration dispersion after a vortex mixer with a constant step}

To study the change in the quality of mixing in a circular pipe after an axial twisting device made in the form of a screw, we examined the average dispersion of concentrations in planes perpendicular to the axis of the apparatus and plot the dependence of the average dispersion on the distance to the screw.

As a calculation model, we took a pipe with a diameter of $0.3 \mathrm{~m}$ with a twisting device made in the form of 2.5 turns of a screw with a step equal to 0.18 and $0.24 \mathrm{~m}$. The mixed components enter the pipe with different speeds through the same pipe cross-sectional area. Based on the results of studies obtained by computer simulation of hydromechanical processes, we plotted the dependences of the average concentration dispersion on the distance to the screw (Figure 4).
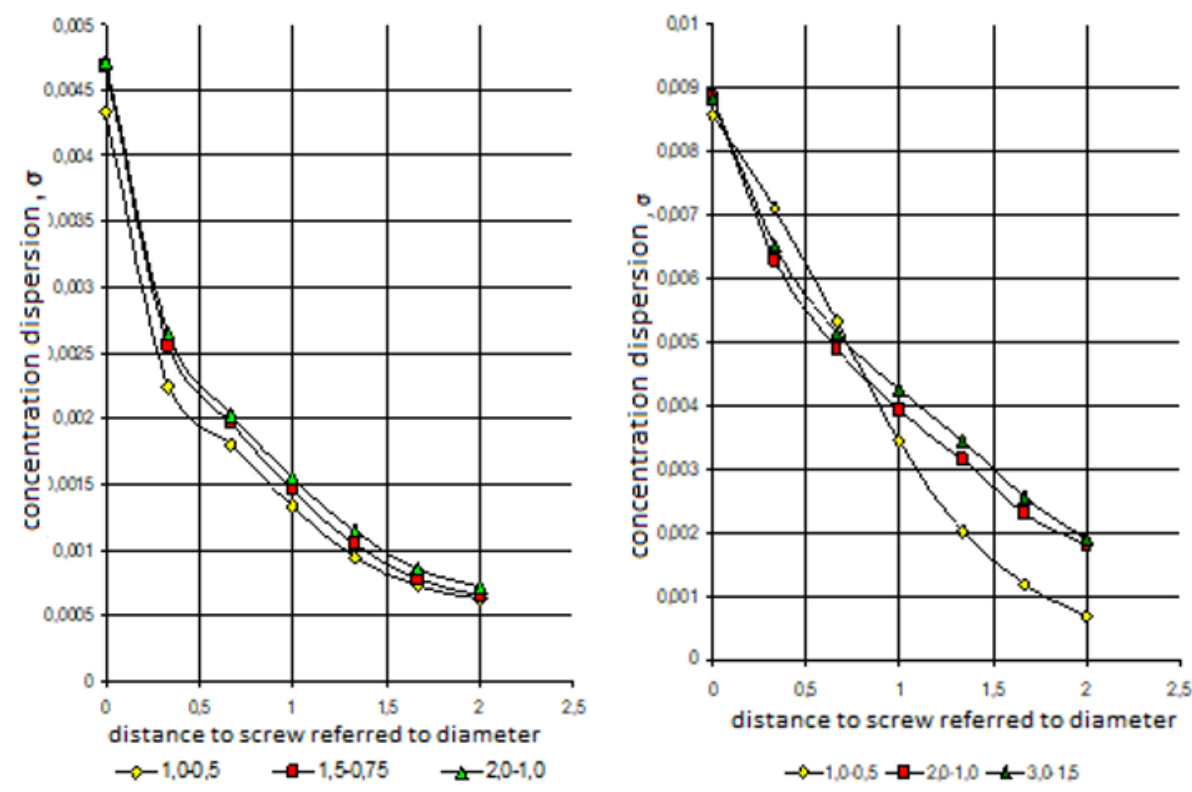

个concentration dispersion, $\rightarrow$ distance to screw referred to diameter auger 2.5 turns long and pitch: a) $180 \mathrm{~mm}$; b) $240 \mathrm{~mm}$

Fig. 4. Graphs of the dependence of the concentration dispersion on the distance to the swirling device at different initial speeds of the mixed components. 
As follows from the obtained dependences, the sharpest increase in the quality of mixing of the components is observed after the screw at a distance of $(0.6 \div 1.0) \mathrm{D}$, and the pattern repeated at different input speeds. This phenomenon can be explained not by the stationary flow of the liquid, which appears when the intensity of the swirling flow changes, and the most abrupt change occurs directly behind the swirling device.

\section{Change in the main characteristics of the mixer when changing the number of turns of the screw}

To determine the dependence of mixing quality when changing the number of turns of the screw with a constant step, the model used was a pipe with a diameter of $300 \mathrm{~mm}$, into which 2 components with speeds of 1 and $0.5 \mathrm{~m} / \mathrm{s}$ were used. The mixing device is an axial twisting device made in the form of a screw with a variable number of turns. The number of turns equal to 1.0 was taken as a minimum (i.e., the cross section of the pipe is completely blocked by the screw), and for the maximum value of the number of turns is 3.0.

We considered the main characteristics of mixing devices with a spool pitch of 180 and 240 mm.

According to the data obtained, a graph of the dependence of resistance (pressure drop) on the number of turns of the screw with a pitch of 180 and $240 \mathrm{~mm}$ was constructed (Figure 5).

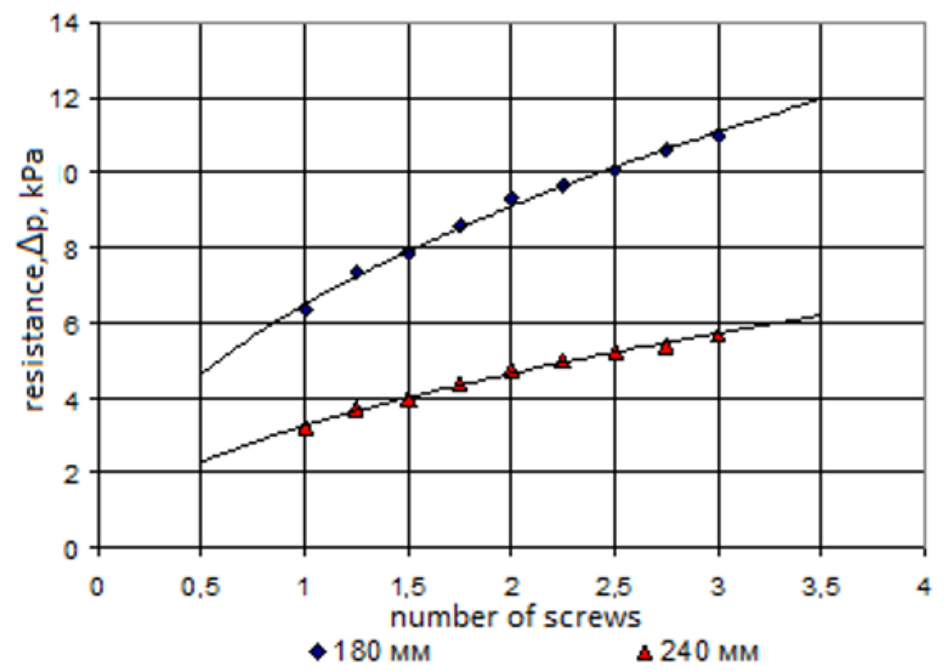

个resistance, $\Delta \mathrm{p}, \mathrm{kPa}, \rightarrow$ number of screws

Fig. 5. Graph of resistance versus the number of turns of the screw

From the obtained dependence it follows that the main pressure drop is created at the first turns of the screw, this can be explained by the fact that it is at the first turns of the screw $(1.0 \div 2.0)$ that the flow is given a swirling movement, and at subsequent turns only the specified twist intensity is maintained.

The concentration dispersion was considered in the cross section of the pipe at a distance of $0.20 \mathrm{~m}$ from the twisting device, since according to the studies conducted, it was at this distance that a sharp increase in the quality of mixing was observed. According to the results obtained in the course of these calculations, we plotted the concentration dispersion versus the number of turns of the screw at a step of 0.18 and $0.24 \mathrm{~m}$ (Figure 6). 


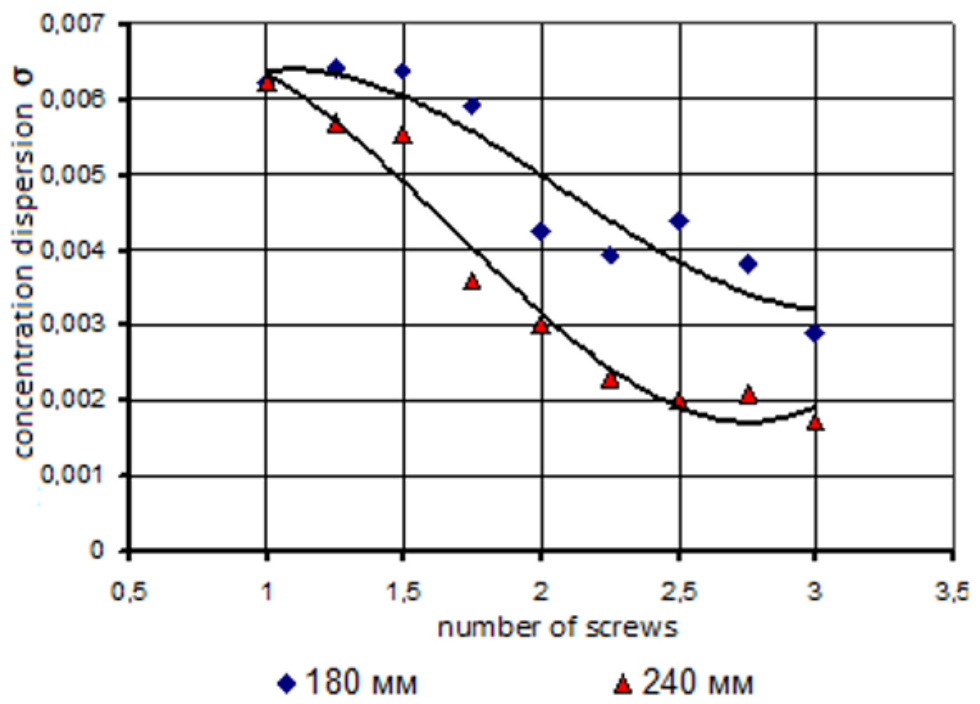

个concentration dispersion, $\rightarrow$ number of screws

Fig. 6. Graph of the concentration dispersion versus the number of turns of the screw in a plane perpendicular to the direction of movement

The obtained data confirmed the assumption that swirling flow is imparted to the first turns of the screw, since it is after $1.5 \div 2.0$ steps of the swirling device that a sharp (38.4 and $58 \%$ increase at steps of 180 and $240 \mathrm{~mm}$ respectively) increase the mixing efficiency after which the growth rate decreases.

\section{Conclusion}

Thus, the use of swirling devices in direct-flow mixers allows to intensify the process without significantly increasing the overall dimensions of the apparatus. Therefore, it is necessary to study in detail the effect of the geometric dimensions of such devices on the main mixing characteristics, for this it is advisable to use computer simulation of hydromechanical processes.

It is also worth noting that the studies of combination a two-component mixture allow us to identify only qualitative dependences of the main characteristics of a direct-flow mixer based on the creation and maintenance of swirling motion, on its geometric dimensions, and do not reflect the entire complexity of the process (effect on the process of physicochemical properties and the amount of incoming components). But, nevertheless, the obtained results should facilitate the development of new designs of direct-flow vortex mixers of increased efficiency.

\section{References}

1. Molokanov Yu.K., Processes and apparatuses for oil and gas refining, (M., Chemistry, 1980)

2. F.S. Khafizov, V.G. Afanasenko, I.F. Khafizov, A.S. Khaibratchmanov, Main Treatment Ways of Manufacturing Water on the Local Plants, Russian journal of applied chemistry, v. 81, №. 9, pp. 1697-1698 
3. Planovsky A. N., Nikolaev P. I., Processes and apparatuses of chemical and petrochemical technology (M., Chemistry, 1972.- 496 p.)

4. Alessandro Paglianti, Giuseppina Montante, A mechanistic model for pressure drops in corrugated plates static mixers, Chemical Engineering Science, v. 97, pp. 376-38, (2013)

5. Kasatkin A. G., Main processes and apparatuses of chemical technology, Ed. 9th. M.: Chemistry, p. 752, (1973)

6. Esau A. Hernandez, Gabriela Sanchez-Reyna, Jorge Ancheyta, Comparison of mixing rules based on binary interaction parameters for calculating viscosity of crude oil blends, Fuel, v. 249, pp. 198-2051, (2019)

7. Kondranin T.V., Tkachenko B.K., Bereznikova M.V., Evdokimov A.V., Zuev A.P. The use of application software packages in the study of fluid and gas mechanics courses: (Textbook, M.: MIPT, 2005)

8. E. Chabanon, N. Sheibat-Othman, O. Mdere, J. P. Valour, F. Puel, Drop size distribution monitoring of oil-in-water emulsions in SMX+ static mixers: Effect of operating and geometrical conditions, International Journal of Multiphase Flow, v. 92, pp. 61-69, (2017)

9. Bin Han, Bao-Wen Yang, and Yudong Zha Numerical study on the effect of grid mixing span in $2 \times 1$ spacer grid, Nuclear Engineering and Design, v. 339, pp. 11-201, (2018)

10. Sister V.G., Martynov Yu.V., The principles of increasing the efficiency of heat and mass transfer processes, Kaluga: Publisher N. Bochkoreva, 508 p., (1998)

11. Afanasenko V.G., Kuzeev I.R., Koushova Y.S., Kulakov P.A., Emulsification intensification under simultaneous impact of original and reflected ultrasonic vibrations, Bulletin of the Tomsk polytechnic university-geo assets engineering, $\mathrm{v}$. 329(11), pp. 44-51Issue: 11

12. Tiezheng Zhao, Xiao Liu, Jialong Yang, Hongtao Zheng, Investigation on effect of mixing distance on mixing process and combustion characteristics of double-cone burner, Fuel, v. 253, pp. 540-551, (2019)

13. V. B. Zametaev, A. R. Gorbushin, I. I. Lipatov, Steady secondary flow in a turbulent mixing layer, International Journal of Heat and Mass Transfer, v. 132, pp. 655-661, (2019)

14. Marie-Luise Maier, Stefanie Milles, Sebastian Schuhmann, Gisela Guthausen, Mathias J. Krause Fluid flow simulations verified by measurements to investigate adsorption processes in a static mixer, Computers \& Mathematics with Applications, v. 76, Issues 11-12, pp. 2744-27571, (2018)

15. K. V. Moiseev, E. V. Volkova, S. F. Urmancheev, Effect of Convection on Polymerase Chain Reaction in a Closed Cell, IUTAM Symposium on Waves in Fluids - Effects of Nonlinearity, Rotation, Stratification and Dissipation, v. 8, pp. 172-175, (2012) 